\title{
Efecto del tipo de activador en un mortero de escoria mezclado con caliza activada alcalinamente
}

\section{Effect of activator type in a slag mortar mixed with alkali-activated limestone}

\author{
DOI: 10.17981/ingecuc.15.2.2019.01 \\ Artículo de investigación científica. Fecha de Recepción: 31/10/2018. Fecha de Aceptación: 14/05/2019 \\ Nelson Virgilio Torres-Rubio \\ Universidad Pedagógica y Tecnológica de Colombia. Tunja (Colombia) \\ nelsonvirgilio.torres@uptc.edu.co \\ Jorge Andrés Sarmiento-Rojas \\ Universidad Pedagógica y Tecnológica de Colombia. Tunja (Colombia) \\ jorge.sarmiento02@uptc.edu.co \\ Oscar Javier Gutiérrez-Junco \\ Universidad Pedagógica y Tecnológica de Colombia. Tunja (Colombia) \\ oscarjavier.gutierrez@uptc.edu.co
}

Para citar este artículo:

N. Torres-Rubio, J. Sarmiento-Rojas y O. Gutiérrez-Junco "Efecto del tipo de activador en un mortero de escoria mezclado con caliza activado alcalinamente", INGE CUC, vol. 15, no. 2, pp. 1-10, 2019. DOI: http://doi.org/10.17981/ingecuc.15.2.2019.01

\section{Resumen}

Introducción- Las escorias activadas alcalinamente han sido una base fundamental en el desarrollo de nuevos cementos con propiedades mecánicas y fisicoquímicas las cuales en algunos casos han sido mejores que las del cemento tradicional, este tipo de productos industriales conlleva al estudio de materiales de construcción alternativos.

Objetivo- Estudiar el efecto de diferentes tipos de activadores alcalinos, adición de caliza y cambios de temperatura sobre las propiedades físico-mecánicas de los morteros a base de escoria activados alcalinamente.

Metodología- Se realiza un análisis fisicoquímico de las características de las materias primas mediante pruebas de MEB, FRX, DRX. Se desarrollaron pruebas preliminares para determinar la dosificación óptima de los activadores utilizados, para luego elaborar los morteros objeto del estudio y evaluar su propiedad mecánica. El estudio se desarrolla para tres soluciones alcalinas $\mathrm{Na}_{2} \mathrm{SiO}_{3} / \mathrm{NaOH}(\mathrm{SH}), \mathrm{Na}_{2} \mathrm{SiO}_{3} / \mathrm{Na}_{2} \mathrm{CO}_{3}$ (SC) y $\mathrm{Na}_{2} \mathrm{SiO}_{3}$ (SS), así como para dos temperaturas de curado a temperatura ambiente $\left(23^{\circ} \mathrm{C}\right)$ y $60^{\circ} \mathrm{C}$.

Resultados- Como resultado se obtuvo el activador con mejor comportamiento, el cual fue el $\mathrm{Na}_{2} \mathrm{SiO}_{3} / \mathrm{NaOH}(\mathrm{SH})$ alcanzando una resistencia de $35,3 \mathrm{MPa}$

Conclusiones- De acuerdo con los resultados, los morteros que presentaron mejor comportamiento fueron aquellos sometidos a temperaturas de $60^{\circ} \mathrm{C}$ y a mayor tiempo de curado aumentando sus propiedades de resistencia.

Palabras clave- Solución alcalina; adición de caliza; MEB (Microscopia Electrónica de Barrido); FRX (Fluorescencia de Rayos X); DRX (Difracción de Rayos X); Resistencia a la compresión; Morteros; Escoria; dosificación óptima.

\begin{abstract}
Introduction- Alkaline activated slag has been a fundamental base in the development of new cements with mechanical and physicochemical properties which in some cases have been better than those of traditional cement, this type of industrial products leads to the study of alternative construction materials.

Objective- To study the effect of different types of alkaline activators, limestone addition and temperature changes on the physical-mechanical properties of alkaline-activated slag-based mortars.
\end{abstract}

Methodology- A physicochemical analysis of the characteristics of the raw materials is carried out by means of tests of SEM, XRF, XRD. Preliminary tests were developed to determine the optimal dosage of the activators used, to later elaborate the mortars object of the study and to evaluate their mechanical property. The study is developed for three alkaline solutions $\mathrm{Na}_{2} \mathrm{SiO}_{3} / \mathrm{NaOH}(\mathrm{SH}), \mathrm{Na}_{2} \mathrm{SiO}_{3} / \mathrm{Na}_{2} \mathrm{CO}_{3}$ (SC) and $\mathrm{Na}_{2} \mathrm{SiO}_{3}$ (SS), as well as for two curing temperatures at room temperature $\left(23^{\circ} \mathrm{C}\right)$ and $60^{\circ} \mathrm{C}$.

Results- As a result, the activator with the best performance was obtained, which was the $\mathrm{Na} 2 \mathrm{SiO} / \mathrm{NaOH}(\mathrm{SH})$ reaching a resistance of $35.3 \mathrm{MPa}$.

Conclusions-According to the results, the mortars that presented better behavior were those submitted to temperatures of $60^{\circ} \mathrm{C}$ and a longer curing time increasing their resistance properties.

Keywords-Alkaline solution; limestone addition; SEM (Scanning Electron Microscopy); XRF (X-Ray Fluorescence); XRD (X-Ray Diffraction); Compression resistance; Mortars; Slag; Optimum dosage. 


\section{INTRODUCCIÓN}

Una realidad presente en todos los sectores de la industria en el mundo es la búsqueda de nuevas tecnologías en pro del desarrollo de actividades que garanticen la sostenibilidad integral de la misma. Teniendo en cuenta esto, y que además la economía está basada en una fuerte industrialización, se ha incrementado el interés por la reutilización de los residuos generados en las diferentes industrias [1].

Una de las industrias de mayor crecimiento a nivel mundial es la industria de la construcción, cuyos insumos principales son el cemento Portland y el acero estructural. Durante el proceso de fabricación de estos insumos se generan toneladas de residuos altamente contaminantes [2].

Según las estadísticas del Departamento Administrativo Nacional de Estadística (DANE) la producción del cemento gris en Colombia para el mes de mayo fue 1 millón 29 mil 400 toneladas, de lo cual se infiere que la producción de $\mathrm{CO}_{2}$ fue de aproximadamente de 863,58 mil toneladas para este año [3].

En el proceso de producción del acero se genera como residuo principal la Escoria de Alto Horno (EAH), la cual se estima generara entre 0,28-0,34 toneladas de escoria por tonelada de acero producida [4]. Y su aprovechamiento genera un impacto positivo al medio ambiente.

La Escoria de Alto Horno (EAH) es un material empleado como insumo para la fabricación de cementos alcalinos, por presentar características que favorecen al desarrollo sostenible de la industria de la construcción, y dichas características son las siguientes:

- Su obtención no genera un elevado costo por ser un subproducto de la producción del acero.

- Su aprovechamiento genera un impacto positivo al medio ambiente.

Los cementos activados alcalinamente son el producto de la mezcla de un mineral de naturaleza amorfa basado en aluminosilicatos (llamado precursor) y un agente alcalino (llamado activador) para iniciar una serie de reacciones que pueden llegar a producir un material con propiedades cementantes. Las propiedades de los cementos activados alcalinamente pueden presentar variaciones, a causa de la calidad de los materiales empleados en el proceso de activación alcalina.

El principio en el cual está basada la activación alcalina fue expuesto [5] en el año de 1949, pero los primeros cementos activados alcalinamente fueron desarrollados por los años 60 en la universidad de Kiev [6], [7]. En las décadas de los 80 y 90 se generó un creciente interés por este tipo de cementos en varios países, como en Europa occidental [8], [9], [10], India [11], Japón [12], China [13], [14] y USA [15].

A partir del análisis de la resistencia adquirida por un cemento alcalino a base de Escoria de Alto Horno (material precursor) y tres diferentes tipos de activadores $\left(\mathrm{Na}_{2} \mathrm{SiO}_{3}, \mathrm{NaOH} / \mathrm{Na}_{2} \mathrm{SiO}_{3}\right.$ y $\left.\mathrm{Na}_{2} \mathrm{CO}_{3} / \mathrm{Na}_{2} \mathrm{SiO}_{3}\right)$, al cual se le adicionó un porcentaje de caliza y se le estudio el cambio de temperatura en el proceso de curado. A continuación, se presentan algunos resultados obtenidos en nuestra investigación con relación a las propiedades de resistencia.

\section{REVISIÓN LITERARIA}

\section{A. Geopolímeros}

Es un término empleado para identificar materiales sintéticos de aluminosilicatos, los cuales son el resultado de la síntesis de una solución alcalina y un material cuya matriz está constituida principalmente por aluminosilicatos.

El termino geopolímero se utilizó por primera vez en la década de los 70 por Davidovits Joseph, para denotar materiales compuestos por sialatos formados por tetraedros de $\mathrm{AlO}_{4} \mathrm{ySiO}_{4}$, los cuales están enlazados con átomos de oxígeno. Para poder dar el balance de la carga de $\mathrm{Al}_{3}+$, son fundamentales los iones positivos

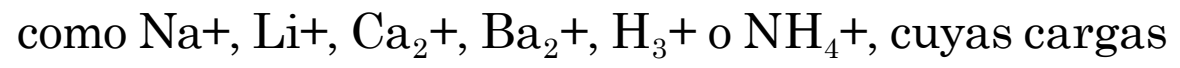
son positivas.

\section{B. Activación alcalina}

Se le conoce como activación alcalina a la solución capaz de formar productos de hidratación de naturaleza adhesiva y cementante. Los materiales activantes pueden ser de naturaleza alcalina o alcalinotérrea, como las sales de ácido fuerte, sales de ácido débil, sales silícicas e hidróxidos.

La activación alcalina de Escoria de Alto Horno (EAH), es un proceso químico en el cual el componente vítreo de este subproducto es convertido en un material cementante.

\section{Primeras investigaciones}

El primer uso del álcali como componente de un material de cementante se remonta a 1930, cuando Kuhl investigó el comportamiento del ajuste de las mezclas del polvo de la escoria granulada y de la solución cáustica de la potasa. Chassevent midió la reactividad de las escorias usando potasa cáustica y una solución de soda caustica en 1937. Purdon realizó el primer estudio extensivo de laboratorio sobre cementos sin Clinker compuestos por escoria y álcalis de soda cáusticos producidos por una base y una sal alcalina en 1940 [16]. 
Hacia finales del año 1957, se producen los primeros materiales ligantes empleando productos como aluminosilicatos (arcillas) y soluciones de metal alcalino con un contenido de calcio muy bajo por el ruso Glukhovsky, quien también propuso que, dependiendo de la composición de las materias primas, los materiales ligantes podrían ser divididos dentro de dos grupos [16].

- Sistemas de unión alcalinos $\mathrm{Me}_{2} \mathrm{O}-\mathrm{Me}_{2} \mathrm{O}_{3}-\mathrm{SiO}_{2}$ $\mathrm{H}_{2} \mathrm{O}$.

- Sistemas de unión álcali alcalino-térreos $\mathrm{Me}_{2} \mathrm{O}$ $\mathrm{MeO}-\mathrm{Me}_{2} \mathrm{O}_{3}-\mathrm{SiO}_{2}-\mathrm{H}_{2} \mathrm{O}$.

Amplias investigaciones han permitido el desarrollo de cementos y concretos activados alcalinamente desde entonces. Algunos de las cuales fueron desarrollados por Cementos Trief y los Cementos $F$ desarrollados en los países Escandinavos Forss y las mezclas de activadores alcalinos con cementos son algunos ejemplos [16].

En 1981, el francés Joseph Davidovits sintetizó algunos materiales ligantes mediante una mezcla álcalis con caolinita calcinada, caliza y dolomitas. El introduce por primera vez el termino geopolímero para llamar a los materiales ligantes poseen una estructura polimérica. También usó varias marcas registradas tales como Pyrament, Geopolycem y Geopolymite para identificar los materiales ligantes. Estos tipos de materiales tienen un comportamiento similar a los materiales alcalinos y prácticamente pertenecen a los sistemas de unión alcalina $\mathrm{Me}_{2} \mathrm{O}$ $\mathrm{Me}_{2} \mathrm{O}_{3}-\mathrm{SiO}_{2}-\mathrm{H}_{2} \mathrm{O}$ [16].

En 1986, se determina que algunos tipos de cemento procedentes de la activación alcalina tienen una matriz solida constituida por desechos radioactivos. En tanto, hicieron la apreciación sobre algunos cementos antiguos que presentaban características similares con dichos materiales [16].

En el año de 1994 se demuestra como los álcalis y las sales de metales alcalinos, así como también los silicatos, aluminatos y aluminosilicatos, reaccionan en una solución alcalina cuando la concentración de esta es suficiente para generarla. Esta interacción tiene lugar con minerales de arcilla, con cristales de aluminosilicatos de origen natural y artificial, en los cuales no hay presencia de calcio, así como con sistemas de fijación de calcio en condiciones naturales, y así se puede formar un hidroaluminosilicato alcalino o alcalinotérreo endurecido resistente al agua, semejantes a las zeolitas y micas naturales [22].

Estudios realizados en 2007, evaluaron las propiedades mecánicas y de durabilidad de morteros de escoria activada alcalinamente, encontrando una elevada estabilidad en estos, tanto con waterglass como con $\mathrm{NaOH}$, en medios sulfáticos y en agua de mar [2].
En el año 1997 se determina un valor umbral de concentraciones en torno al $4 \%$ en peso de $\mathrm{NaO}_{2}$ y en dichas concentraciones a las temperaturas de ensayo (excepto a $25^{\circ} \mathrm{C}$ con un $3 \%$ en peso de $\mathrm{NaO}_{2}$ ), con energía de activación obtenida del orden de 50-58 kJ/ mol [17].

Para el 2009 se determinó que el contenido de silicatos solubles presentes en el activador alcalino, tiene un efecto importante en la cinética de la reacción de geopolimerización, características micro-estructurales del producto formado, así como en las propiedades físico-mecánicas [18].

En 2011 se realizaron estudios a morteros a base de vidrio de desecho/escoria de alto horno y a la activación mecano química del vidrio en soluciones y determinaron como la resistencia a la compresión de los morteros de 100\% escoria disminuia al aumentar el contenido de $\mathrm{Na}_{2} \mathrm{O}$ y disminuia con el curado a $60^{\circ} \mathrm{C}$. En contraste, los cementos $100 \%$ Vidrio de Desecho (VD) requirieron condiciones químicamente más agresivas $\left(12 \% \mathrm{Na}_{2} \mathrm{O}\right.$ y curado a $\left.60^{\circ} \mathrm{C}\right)$ para obtener buenas propiedades mecánicas [19].

\section{Metodologia}

Para este trabajo se plantea el procedimiento experimental en cada una de las etapas, incluyendo la preparación mecánica de los materiales empleados, caracterización y las pruebas preliminares realizadas con el fin de determinar la dosificación adecuada para los morteros de escoria.

Se utilizó como material precursor escoria granulada de alto horno proveniente de acerías paz del rio (Belencito, Boyacá), con granulometría de $75 \mu \mathrm{m}$. La composición química de dicha escoria se observa en la Tabla I.

TABLA I. COMPOSICIÓN QUímiCA DE LA ESCORIA.

\begin{tabular}{|c|c|c|c|c|c|}
\hline Componente & $\mathrm{Al}$ & $\mathrm{Si}$ & $\mathrm{S}$ & $\mathrm{Ca}$ & $\mathrm{Mn}$ \\
\hline$\%$ en peso & $14,2 \%$ & $22,4 \%$ & $1,5 \%$ & $56,2 \%$ & $2,3 \%$ \\
\hline
\end{tabular}

Fuente: Autores.

También se utilizó caliza proveniente de una cantera del municipio de Villa de Leiva (Boyacá), la cual tuvo una serie de operaciones mecánicas para permitir la reducción de tamaño y separación de partículas con la siguiente composición química (Tabla II).

TABla II. Composición QUÍMICA DE LA CALIZA.

\begin{tabular}{|c|c|c|c|c|c|c|}
\hline Componente & $\mathrm{Na}$ & $\mathrm{Al}$ & $\mathrm{Si}$ & $\mathrm{K}$ & $\mathrm{Ca}$ & $\mathrm{Fe}$ \\
\hline \% en peso & 9.9 & 5 & 7.2 & 1.3 & 68 & 7.6 \\
\hline
\end{tabular}

Fuente: Autores. 
La composición de las soluciones alcalinas empleadas para la realización de este trabajo se presentan en la Tabla III. Estas soluciones se prepararon con una mezcla comercial de Silicato de sodio $\left(\mathrm{Na}_{2} \mathrm{SiO}_{3}\right)$, Carbonato de sodio $\left(\mathrm{Na}_{2} \mathrm{CO}_{3}\right)$ e Hidróxido $(\mathrm{NaOH})$ y agua destilada. Con estas soluciones se prepararon mezclas preliminares donde se evaluaba el comportamiento a la compresión a diferentes que iban en edades de 3, 7 y 28 días de pastas con tres diferentes porcentajes de activadores al 40\%, 50\% y 60\% para los tres activadores propuestos. Estas pastas fueron evaluadas con dos temperaturas de curado $\left(60^{\circ} \mathrm{C}\right.$ y temperatura ambiente $23^{\circ} \mathrm{C}$ ).

El curado se realizó mediante el método "cured in covered molds" [20].

Tabla III. Porcentajes de soluciones.

\begin{tabular}{|c|c|c|c|c|}
\hline Activador (\%) & $\mathrm{Na}_{2} \mathrm{SiO}_{3} \%$ & $\mathrm{NaOH} \%$ & $\mathrm{Na}_{2} \mathrm{CO}_{3} \%$ & $\mathrm{H}_{2} \mathrm{O} \%$ \\
\hline \multirow{4}{*}{40} & 61,5 & - & - & 38,5 \\
\cline { 2 - 5 } & 26,7 & - & 26,7 & 46,7 \\
\cline { 2 - 5 } & 28,6 & 28,6 & - & 42,9 \\
\hline \multirow{4}{*}{50} & 66,7 & - & - & 33,3 \\
\cline { 2 - 5 } & 29,4 & - & 29,4 & 41,2 \\
\cline { 2 - 5 } & 31,3 & 31,3 & - & 37,5 \\
\hline \multirow{3}{*}{60} & 58,3 & - & - & 41,7 \\
\cline { 2 - 5 } & 28,9 & - & 28,9 & 58,3 \\
\cline { 2 - 5 } & 25,0 & 25,0 & - & 50,0 \\
\hline
\end{tabular}

Fuente: Autores.

Con los resultados obtenidos de las pastas se evaluó cual era el sistema con el mejor comportamiento a la compresión y a este se le realizó la prueba de DRX con el fin de observar las fases cristalinas presentes en la reacción.

Tabla IV. Resultados de las pastas a temperatura ambiente.

\begin{tabular}{|c|c|c|c|}
\hline \multirow{2}{*}{ Activador } & \multicolumn{3}{|c|}{ Resistencia en Mpa } \\
\cline { 2 - 4 } & 3 días & 7 días & 28 días \\
\hline SC $60 \%$ & 15,8 & 17,7 & 17,4 \\
\hline SH $60 \%$ & 4,5 & 9,8 & 17,5 \\
\hline SS $40 \%$ & 0 & 0 & 1 \\
\hline
\end{tabular}

Fuente: Autores

Habiendo obtenido los resultados de la experimentación preliminar se determina el porcentaje óptimo de activador, y se encuentra que estos eran de $60 \%$ $\mathrm{SH}, 60 \% \mathrm{SC}$ y $40 \%$ SS cuando el proceso de curado se lleva a cabo a temperatura ambiente (se presenta en la Tabla IV). Y 50\% SH, 60\% SC y 40\% SS cuando el proceso de curado se llevó a cabo a $60^{\circ} \mathrm{C}$. (Observar Tabla V).

Tabla V. Resultados de las Pastas a temperatura $60^{\circ} \mathrm{C}$.

\begin{tabular}{|l|c|c|c|}
\hline \multirow{2}{*}{ Activador } & \multicolumn{3}{|c|}{ Resistencia en Mpa } \\
\cline { 2 - 4 } & 3 días & 7 días & 28 días \\
\hline SC $60 \%$ & 7,6 & 10,6 & 12,5 \\
\hline SH $50 \%$ & 10,7 & 10,9 & 11,6 \\
\hline SS $40 \%$ & 1,6 & 1,7 & 1,8 \\
\hline
\end{tabular}

Fuente: Autores.

Con los porcentajes óptimos de activador se establece la relación de agua/escoria+activador para la preparación de las soluciones alcalinas empleadas en la fabricación de los morteros activados alcalinamente con y sin adición de caliza para los resultados obtenidos a partir del ensayo de fluidez, como se presentan en la Tabla VI.

TABLA VI. Relación Agua / (ESCORIA + ACTIVADOR).

\begin{tabular}{|c|c|c|}
\hline Activador & \% EAH & W/E \\
\hline \multirow{2}{*}{ Silicato/Carbonato } & 100 & 0,53 \\
\cline { 2 - 3 } & 90 & 0,53 \\
\hline \multirow{2}{*}{ Silicato/Hidróxido } & 100 & 0,25 \\
\cline { 2 - 3 } & 90 & 0,25 \\
\hline \multirow{2}{*}{ Silicato de sodio } & 100 & 0,23 \\
\cline { 2 - 3 } & 90 & 0,23 \\
\hline
\end{tabular}

Fuente: Autores.

Con estos resultados se procede a elaborar los morteros activados alcalinamente siguiendo parcialmente los lineamientos de la norma NTC220 [21] (determinación de la resistencia de morteros de cemento hidráulico usando cubos de $50 \mathrm{~mm}$ ). El curado de estos morteros se lleva a cabo con el método "cured in covered molds". La resistencia a la compresión de los morteros se determina para tres edades diferentes de 3, 7 y 28 días.

Además, con los resultados obtenidos se determina el sistema que presenta las propiedades mecánicas más elevadas y a este se le realiza la prueba de Microscopia Electrónica de Barrido (MEB) con el fin de observar su morfología.

Con el fin de realizar un completo análisis comparativo, a los morteros de cemento fabricados, se les realiza un proceso de curado a una temperatura ambiente de $23^{\circ} \mathrm{C}$ y a $60^{\circ} \mathrm{C}$. Para la fabricación de estos también se tuvo en cuenta la NTC220 [21]. 


\section{Resultados}

\section{A. Caracterización de materias primas}

\section{1) Análisis de la escoria por difracción de rayos $X$}

En la Tabla VII se presentan los resultados obtenidos mediante DRX. Se observa la presencia de muy poca fase cristalina y una alta naturaleza amorfa que favorece su capacidad reactiva de la escoria. La baja intensidad en las señales en el difractograma Fig. 1, indican la presencia de un alto contenido de material amorfo del $97.1 \%$.

Tabla VII. Compuestos identificados.

\begin{tabular}{|c|c|c|c|}
\hline Muestra & Graphite & Gehlenite & Contenido amorfo \\
\hline EAH & $1.3 \%$ & $1.5 \%$ & $97.1 \%$ \\
\hline
\end{tabular}

Fuente: Autores.

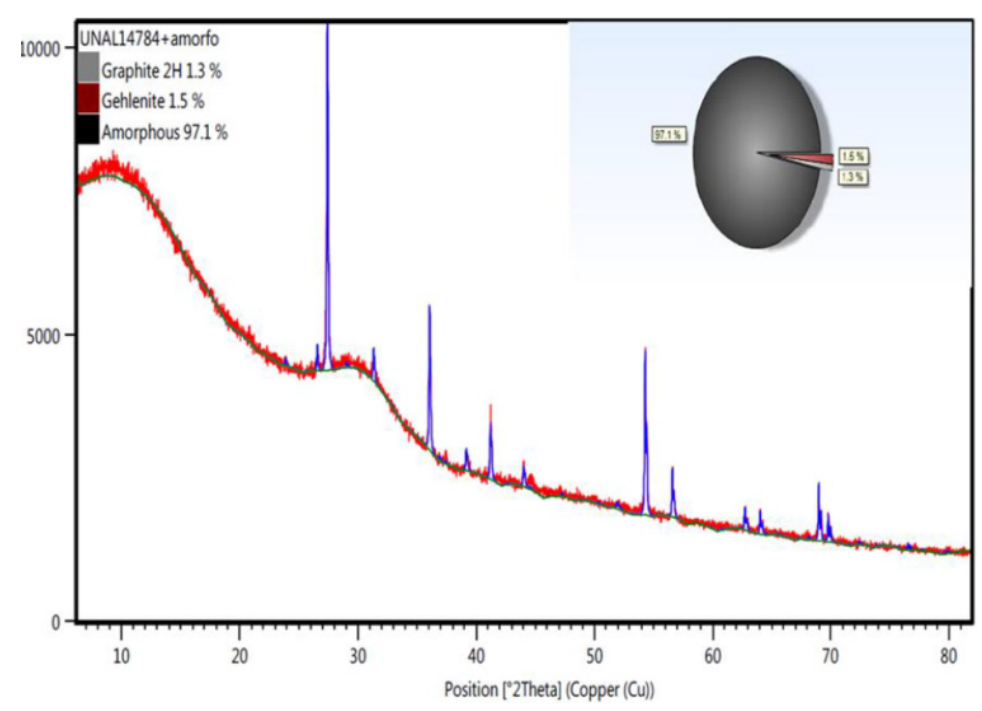

Fig. 1. Difractograma de la escoria.

Fuente: Autores.

\section{2) Fluorescencia de Rayos X para la escoria}

Esta escoria se puede clasificar como una escoria básica al tener una relación $\mathrm{CaO} / \mathrm{SiO}_{2}$ superior a 1 , lo cual es bueno en los procesos de reactividad.

TABla VIII. Compuestos identificados.

\begin{tabular}{|c|c|}
\hline Componente & \% en peso \\
\hline $\mathrm{Mg}$ & $1 \%$ \\
\hline $\mathrm{Al}$ & $14,2 \%$ \\
\hline $\mathrm{Si}$ & $22,4 \%$ \\
\hline $\mathrm{S}$ & $1,5 \%$ \\
\hline $\mathrm{Ca}$ & $56,2 \%$ \\
\hline $\mathrm{Ti}$ & $0,34 \%$ \\
\hline $\mathrm{Mn}$ & $2,25 \%$ \\
\hline $\mathrm{Fe}$ & $1,72 \%$ \\
\hline
\end{tabular}

Fuente: Autores

\section{3) Microscopia Electrónica de Barrido de la escoria.}

Muestras selectas de escoria fueron analizadas para llevar a cabo un estudio microestructura mediante electrones retrodispersados, incluyendo el análisis químico de los productos de reacción (EDS)mediante espectroscopia por dispersión de energía.

Las micrografías MEB son presentadas en la Fig. 2. Se observa que la escoria sin activar presenta en su mayoría morfología angular irregular en su matriz. En el análisis mediante Espectroscopía por Dispersión de Energía (EDS) se realizaron mapeos elementales de $\mathrm{Si}, \mathrm{Al}$, $\mathrm{Na}$ y $\mathrm{Ca}$, con la finalidad de apreciar la distribución de dichos elementos en la microestructura.

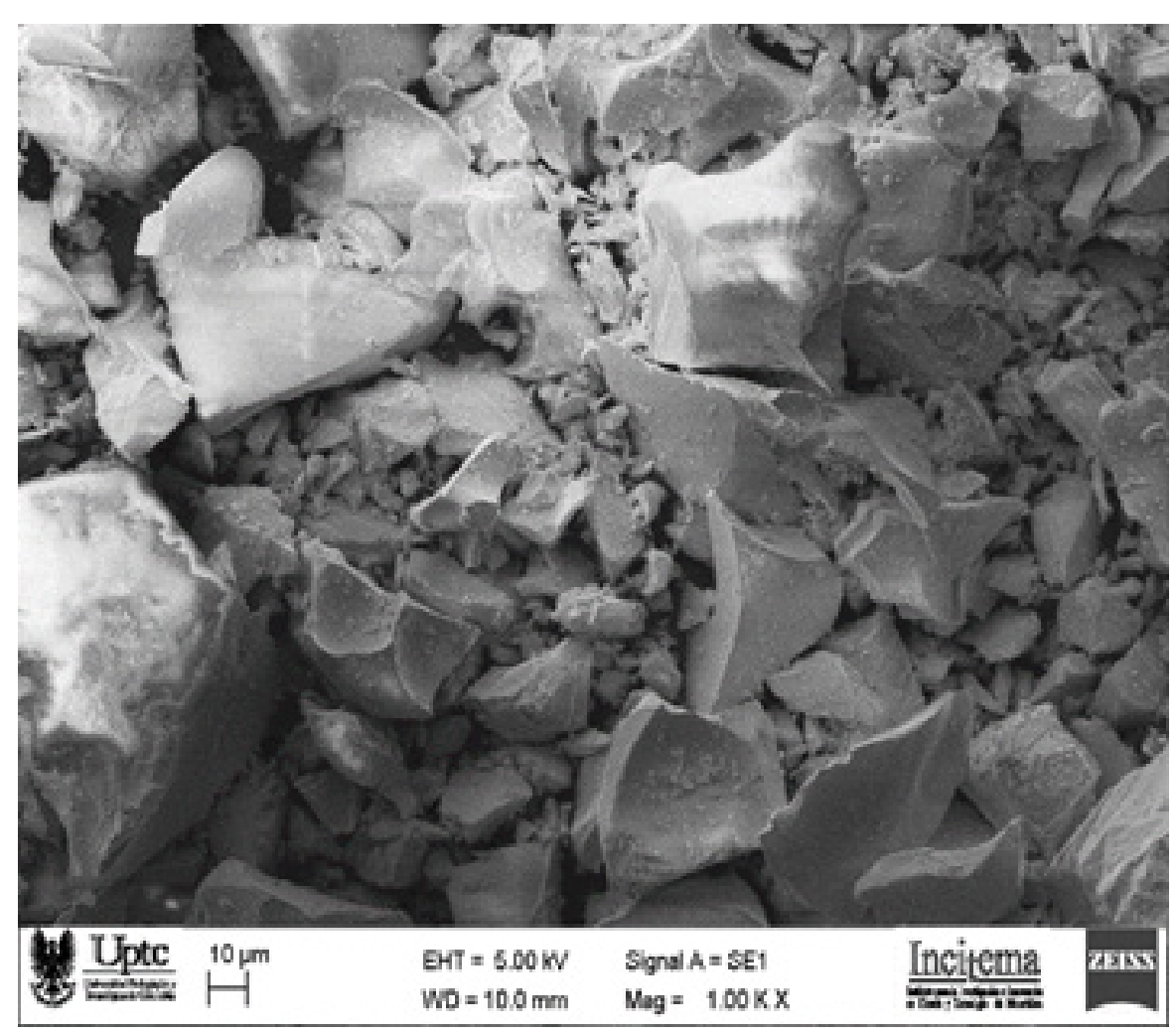

Fig. 2. Micrografía MEB de la escoria.

Fuente: Autores.

\section{4) Fluorescencia de Rayos X para la caliza.}

Se puede observar en la Tabla II los resultados de FRX para la caliza, dándonos a conocer que la caliza de Villa de Leyva (vereda pedrera) contiene una cantidad de $68 \%$ de Ca y un alto contenido de Carbonato de calcio $\left(\mathrm{CaCO}_{3}\right)$.

\section{5) Análisis Termogravimétrico para la caliza}

Se realiza un análisis de TGA y DSC para una muestra base de $20 \mathrm{mg}$. de la mina y se lleva hasta una temperatura de 1.400 para ver su comportamiento en cada una de las temperaturas de reacción de los principales elementos encontrados con el Ensayo de Fluorescencia de Rayos X, como el Calcio, Azufre, Sílice, entre otros, obteniendo los siguientes resultados (Fig. 3). 


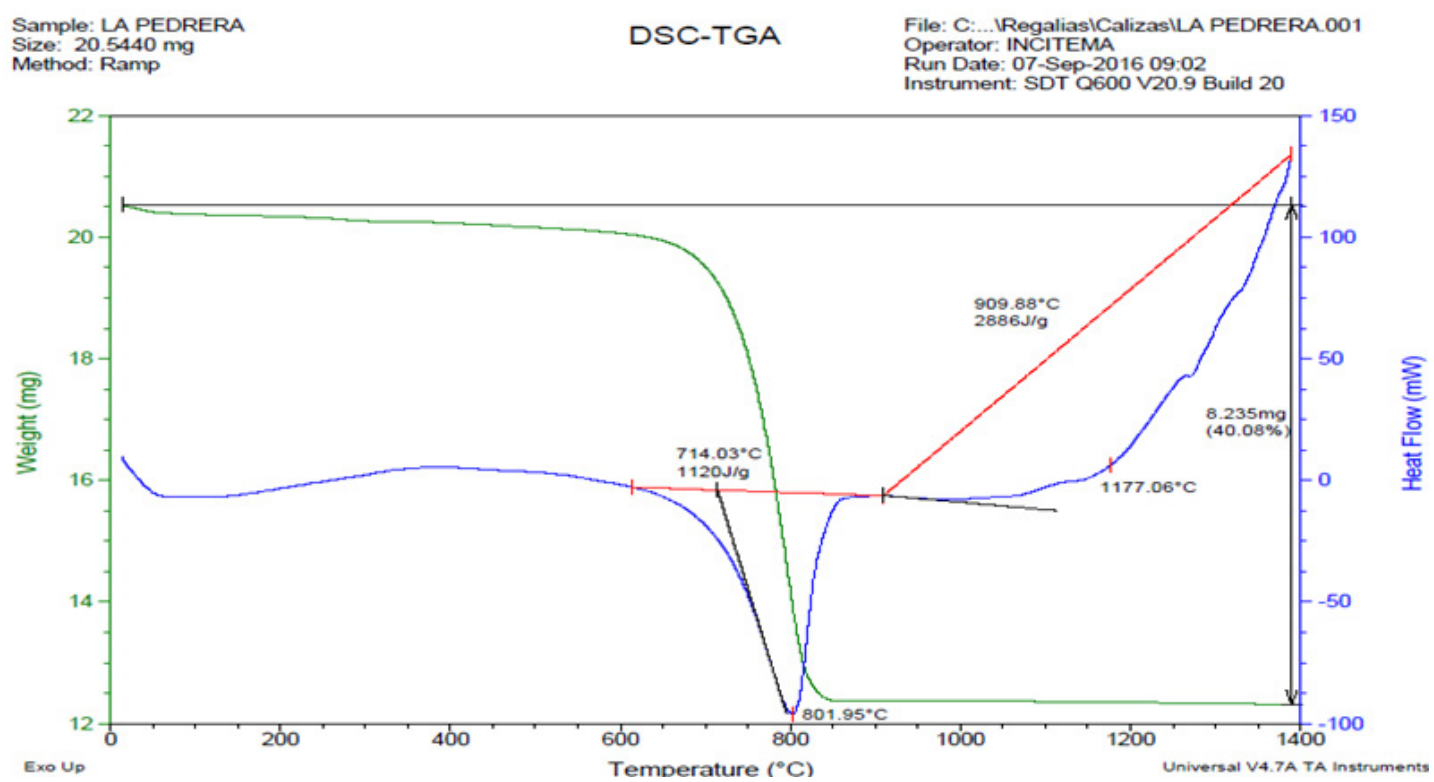

Fig. 3. Curvas TGA y DSC para la piedra caliza.

Fuente: Autores

Se observa en la curva de TGA, una pérdida constante del agua superficial $\left(0-200^{\circ} \mathrm{C}\right.$ aprox.) y molecular (200- $450^{\circ} \mathrm{C}$ aprox.) además de la liberación de Dióxido de carbono $\left(\mathrm{CO}_{2}\right)$ y de Dióxido de azufre $\mathrm{SO}_{2}$, en temperaturas similares a las de salida del agua molecular (hacia los 650 aprox.). También se observa una perdida excesiva de material, llegando así a un proceso de calcinación o transformación de $\mathrm{CaCO}_{3}$, en $\mathrm{CaO}$.

En la curva de DSC, se puede observar como en el proceso de calcinación (650-800 aprox.) se tiene en términos termodinámicos, procesos endotérmicos, como se explica en el proceso anterior, y se distingue un promedio de aumento en la temperatura.

\section{6) Microscopia Electrónica de Barrido para la caliza}

Se analizaron muestras de tamaño de grano pasante tamiz No. 200 (tamaño de partícula $\leq 75 \mu \mathrm{m}$ ) de la mina especificada anteriormente.

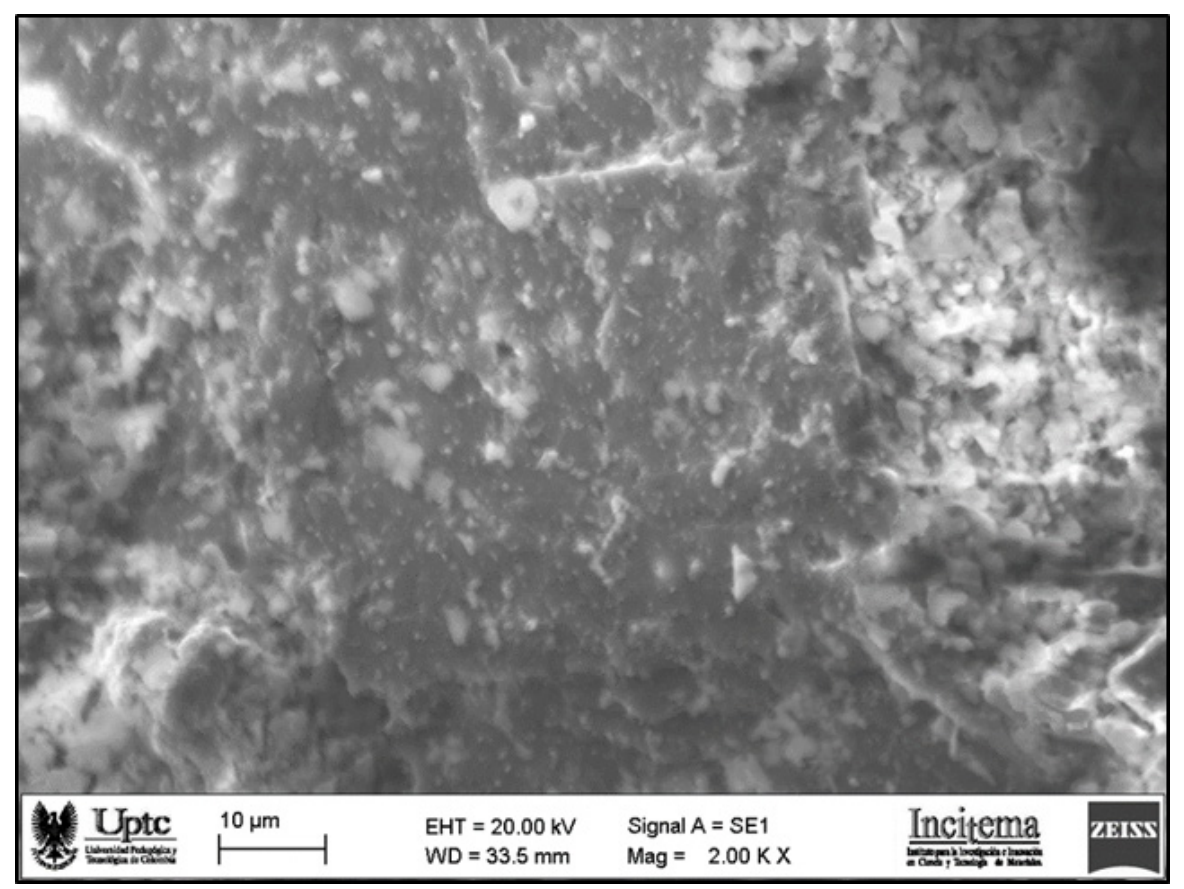

Fig. 4. Micrografía MEB. Fuente: Autores.
En la Fig. 4 se observa como las intrusiones de calcita $\left(\mathrm{CaCO}_{3}\right)$ tienen tono de color blanco, mientras que los demás materiales como el Óxido de carbono (CO) y el Dióxido de azufre $\left(\mathrm{SO}_{2}\right)$ presentan tonalidades mas oscuras.

\section{B. Resultados de Resistencia a la compresión de pastas}

Los resultados de resistencia a la compresión obtenidos en las pastas con un curado a temperatura ambiente $\left(23^{\circ} \mathrm{C}\right)$ se presentan en la Tabla VI. Se resalta como el porcentaje de los activadores influye directamente en la resistencia mecánica a la compresión.

TABla IX. RESISTENCIA A LA COMPREsión DE PASTAS CURADAs A TEMPERATURA AMBIENTE $23^{\circ} \mathrm{C}$.

\begin{tabular}{|c|c|c|c|}
\hline \multicolumn{4}{|c|}{ Activadores al $40 \%$} \\
\hline \multirow{2}{*}{ Activador } & \multicolumn{3}{|c|}{ Resistencia en MPa } \\
\hline & 3 días & 7 días & 28 días \\
\hline $\mathrm{SC}$ & 1,5 & 3,5 & 15,5 \\
\hline $\mathrm{SH}$ & 16 & 14 & 20 \\
\hline SS & 0 & 0 & 1 \\
\hline \multicolumn{4}{|c|}{ Activadores al 50\% } \\
\hline \multirow{2}{*}{ Activador } & \multicolumn{3}{|c|}{ Resistencia en $\mathrm{MPa}$} \\
\hline & 3 días & 7 días & 28 días \\
\hline $\mathrm{SC}$ & 9,6 & 13 & 12,1 \\
\hline $\mathrm{SH}$ & 5,2 & 10 & 13,5 \\
\hline SS & 0 & 0 & 0,8 \\
\hline \multicolumn{4}{|c|}{ Activadores al $60 \%$} \\
\hline \multirow{2}{*}{ Activador } & \multicolumn{3}{|c|}{ Resistencia en $\mathrm{MPa}$} \\
\hline & 3 días & 7 días & 28 días \\
\hline $\mathrm{SC}$ & 15,5 & 17,7 & 17,4 \\
\hline $\mathrm{SH}$ & 4,5 & 9,8 & 17,5 \\
\hline SS & 0 & 0 & 0,8 \\
\hline
\end{tabular}

Fuente: Autores. 
Los resultados obtenidos de los sistemas de pastas que presentaron mejor comportamiento fueron $\mathrm{SH}$ al $60 \%$ y SC al 60\%. En relación con el aumento de SS se observa como se reduce la resistencia.

En la Fig. 5 se evidencia como la resistencia a la compresión de las pastas varía dependiendo del tipo de activador y su concentración, mostrando que el activador de menor comportamiento con respecto a la resistencia a la compresión es el elaborado teniendo como base activadora el SS, de igual manera evidencia como los activadores de $\mathrm{SC}$ y $\mathrm{SH}$ presentan una resistencia a la compresión similar a los 28 días. Cabe resaltar que las pastas preparadas con $\mathrm{SC}$, adquirieron una resistencia del $90,8 \%$ a los 3 días, comparado con su resistencia máxima a los 28 días.

Por otro lado, se observa como las pastas de SS adquirieron resistencia hasta una edad de 28 días.

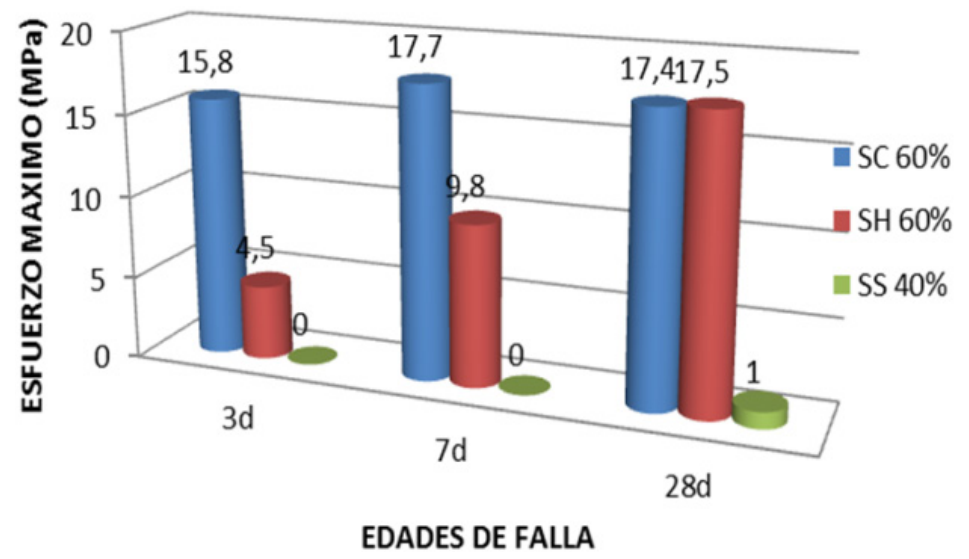

Fig. 5. Resistencia a la compresión de pastas curadas a temperatura ambiente $23^{\circ} \mathrm{C}$.

Fuente: Autores.

TABla X. Resistencia a La COMPRESIÓN DE PASTAS CURADAS A $60^{\circ} \mathrm{C}$.

\begin{tabular}{|c|c|c|c|}
\hline \multirow{4}{|c|}{ Activadores al $40 \%$} \\
\hline \multirow{2}{*}{ Activador } & \multicolumn{3}{|c|}{ Edades de falla } \\
\cline { 2 - 4 } & 3 días & 7 días & 28 días \\
\hline SC & 21 & 5 & 4,8 \\
\hline SH & 8,5 & 9,8 & 10,6 \\
\hline SS & 1,6 & 1,7 & 1,8 \\
\hline \multicolumn{4}{|c|}{ Activadores al $50 \%$} \\
\hline \multirow{3}{*}{ Activador } & \multicolumn{3}{|c|}{ Edades de falla } \\
\cline { 2 - 4 } & 3 días & 7 días & 28 días \\
\hline SC & 8,3 & 14,1 & 12,9 \\
\hline SH & 10,7 & 10,9 & 11,6 \\
\hline SS & 1,6 & 1,1 & 1,5 \\
\hline \multicolumn{4}{|c|}{ Activadores al $60 \%$} \\
\hline \multirow{2}{*}{ Activador } & \multicolumn{3}{|c}{ Edades de falla } \\
\cline { 2 - 4 } & 3 días & 7 días & 28 días \\
\hline SC & 7,6 & 10,6 & 12,5 \\
\hline SH & 5,8 & 4,8 & 4,4 \\
\hline SS & 1,2 & 1,2 & 0,8 \\
\hline
\end{tabular}

Fuente: Autores.
Las pastas SC presentaron resistencias de hasta 21 MPa a los 3 días con un porcentaje de activador del $40 \%$, pero como se evidencia en la Tabla VII esta resistencia decae a los 7 y 28 días con tendencia a continuar disminuyendo. Las pastas de $\mathrm{Na}_{2} \mathrm{SiO}_{3} / \mathrm{NaOH}$ (SH) presentaron su mejor comportamiento cuando el porcentaje de activador fue del $50 \%$, y las pastas de $\mathrm{Na}_{2} \mathrm{SiO}_{3}$ (SS) tienen un comportamiento similar a las pastas curadas a temperatura ambiente.

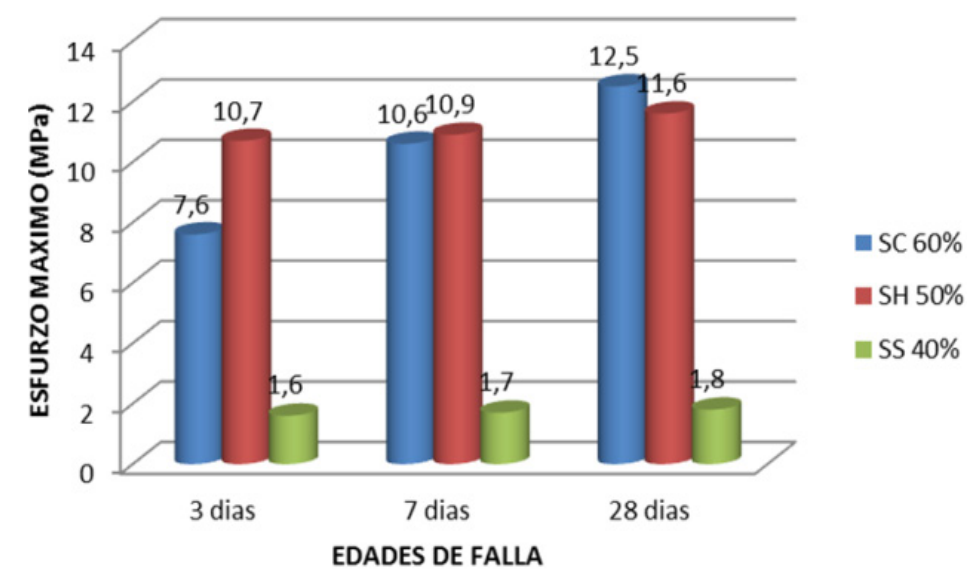

Fig. 6. Resistencia a la compresión de pastas curadas a $60^{\circ} \mathrm{C}$. Fuente. Autores.

Las pastas con mejor comportamiento a la compresión son las $\mathrm{Na}_{2} \mathrm{SiO}_{3} / \mathrm{Na}_{2} \mathrm{CO}_{3}$ (SC) al 60\% (Fig. 6), presentando un aumento progresivo en su resistencia, mientras la relación SH presenta una disminución en la resistencia con un valor aproximadamente de 11,6 Mpa.

\section{Resultados de DRX en las pastas}

Los resultados de caracterización mediante DRX para el sistema preparado de las pastas de escoria se muestra en la Fig. 8 con 28 días de curado, correspondiente al sistema $\mathrm{Na}_{2} \mathrm{O}_{3} \mathrm{Si} / \mathrm{Na}_{2} \mathrm{CO}_{3}$, el cual obtiene una mayor resistencia en las pruebas preliminares.

La Fig. 7 muestra un único cambio notorio observable después de la reacción, el cual es un pico correspondiente a andradita, mineral del grupo de silicatos de hierro y calcio de una típica forma isométrica en sus cristales.

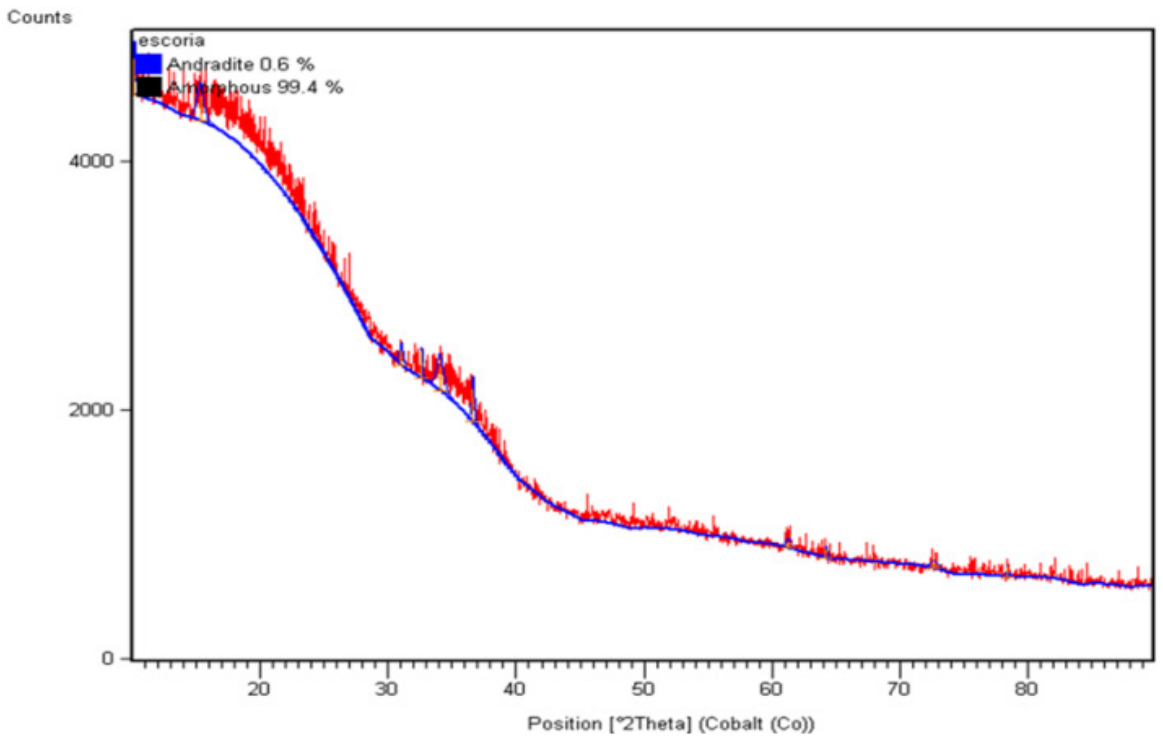

Fig. 7. DRX de pasta de escoria $\mathrm{SC}$ a 28 días. Fuente: Autores. 
Se identifica una reorganización estructural del componente vítreo del material como consecuencia del ataque alcalino con $\mathrm{Na}_{2} \mathrm{O}_{3} \mathrm{Si} / \mathrm{Na}_{2} \mathrm{CO}_{3}(\mathrm{SC})$.

\section{Resistencia a la compresión de los morteros}

Los resultados obtenidos mediante el ensayo de compresión se presentan en la Tabla XI y se pueden observar en la Fig. 8 y Fig. 9. Los sistemas que contenían mayor porcentaje de escoria de alto horno arrojaron los mejores resultados.

TABLA XI. Resistencia A la COMPRESIÓN DE MORTERos ACTIVAdOS ALCALINAMENTE.

\begin{tabular}{|c|c|c|c|}
\hline $\begin{array}{c}\text { Morteros de escoria activados alcalinamente temperatura } \\
\text { ambiente }\left(23^{\circ} \mathrm{C}\right)\end{array}$ \\
\hline \multirow{2}{*}{ Activador } & \multicolumn{3}{|c|}{ Resistencia Mpa } \\
\cline { 2 - 4 } & 3días & 7 días & 28 días \\
\hline SC 60\% & 0,6 & 0,9 & 1,3 \\
\hline SC + Caliza & 0,4 & 0,7 & 1,0 \\
\hline SH & 5 & 14,4 & 32,4 \\
\hline SH + Caliza & 1,6 & 5,2 & 27,6 \\
\hline SS & 0,2 & 0,2 & 0,2 \\
\hline SS + Caliza & 0,2 & 0,1 & 0,2 \\
\hline
\end{tabular}

Morteros de escoria activados alcalinamente temperatura $60^{\circ} \mathrm{C}$

\begin{tabular}{|c|c|c|c|}
\hline \multirow{2}{*}{ Activador } & \multicolumn{3}{|c|}{ Resistencia MPa } \\
\cline { 2 - 4 } & 3días & 7 días & 28días \\
\hline SC 60\% & 1,9 & 2,2 & 5,1 \\
\hline SC + Caliza & 0,7 & 0,8 & 1,45 \\
\hline SH & 32,9 & 30,1 & 35,3 \\
\hline SH + Caliza & 27,7 & 28,5 & 31,1 \\
\hline SS & 0,4 & 0,4 & 0,6 \\
\hline SS + Caliza & 0,4 & 0,3 & 0,3 \\
\hline Morteros convecionales temperatura ambiente $\left(23^{\circ} \mathrm{C}\right)$ \\
\hline \multirow{4}{|c|}{ Resistencia MPa } \\
\hline \multirow{4}{*}{ Cemento tipo UG } & 3 días & 7 días & 28 días \\
\cline { 2 - 4 } & 14,8 & 21,7 & 30,7 \\
\hline
\end{tabular}

Fuente: Autores.

Se observa como la resistencia a la compresión en los morteros activados alcalinamente no aumenta con el pasar del tiempo en todos los morteros, como si lo hace el cemento gris. Los sistemas alcalinos curados a temperatura ambiente no alcanzan una resistencia a los 3 días superior a la alcanzada por el cemento portland a la edad 28 días. Sin embargo, se puede ver como el sistema compuesto por EAH-SH y expuesto a un curado inicial de $60^{\circ} \mathrm{C}$ logra superar dichas resistencias. Estos resultados son comprensibles, ya que este es un comportamiento típico de los morteros en base escoria por su alto contenido amorfo, el cual permite una mejor reacción.
$\mathrm{Al}$ realizarse el comparativo de las resistencias obtenidas por los morteros alcalinamente activados con $\mathrm{SH}$ y los morteros de cemento gris se establece como los morteros activados tienen una resistencia a la compresión del $29,5 \%$ y para los morteros de $\mathrm{SH}$ a $60^{\circ} \mathrm{C}$, $\mathrm{SH}+$ caliza del $19,9 \%$ superior; esto ocurre porque la reacción en la activación alcalina es superior a la reacción del cemento gris. Estos porcentajes varían si se tiene en cuenta los resultados de la resistencia a la compresión de los sistemas curados a temperatura ambiente.

Los demás sistemas no representan valores representativos al no alcanzar valores significativos las resistencias, por el hecho de no ocurrir la reacción total de la estructura amorfa de la escoria.

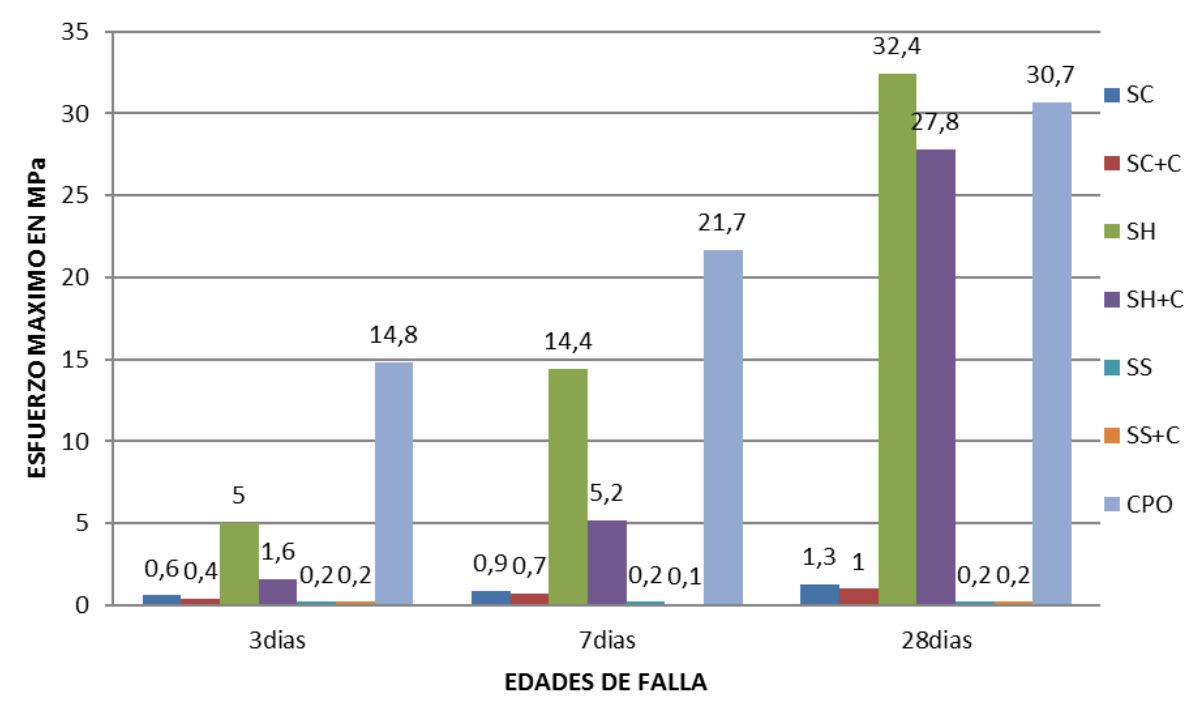

Fig. 8. Resistencia a la compresión morteros curados a temperatura ambienté $\left(23^{\circ} \mathrm{C}\right)$.

Fuente: Autores

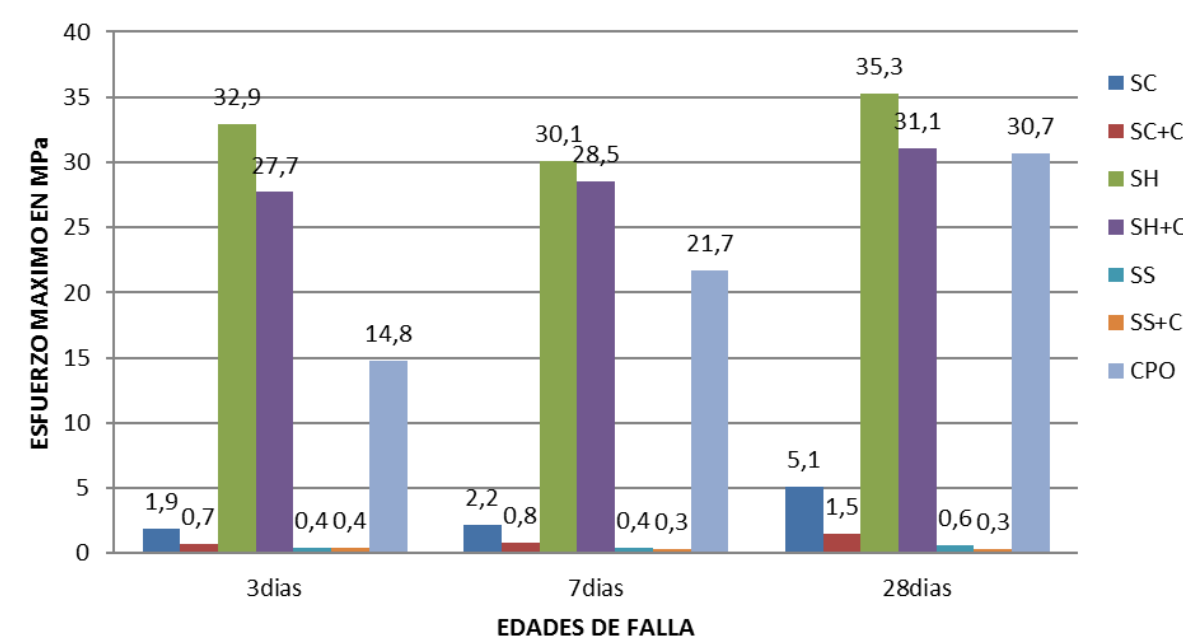

Fig. 9. Resistencia a la compresión morteros curados a temperatura $60^{\circ} \mathrm{C}$.

Fuente: Autores.

\section{E. Resultados de $M E B$}

Luego de haber obtenido los morteros con cada tipo de activador se determina cual es el poseedor de mayor resistencia por medio de los análisis mecánicos y se concluye que el más efectivo es el mortero activado con $\mathrm{Na}_{2} \mathrm{SiO}_{3} / \mathrm{NaOH}$. Estos morteros a una edad de 28 días, se les realiza análisis en el Microscopio 
Electrónico de Barrido (MEB) con fin de observar la distribución de fases y morfología de los granos, es decir la aglomeración de cada uno de sus agregados. Según la Fig. 10 la escoria tiene partículas irregulares y muy afiladas, en cambio la arena muestra una morfología esférica. Las partículas al disolverse forman una matriz que contiene los productos de reacción y continuan en la superficie aquellas partículas que no se disolvieron, formando anillos de reacción en estado sólido alrededor de ellas. De manera general, podemos observar que no aparece porosidad, pero si se detalla la presencia de grietas probablemente creadas por el vacío experimentado dentro de la cámara del microscopio.

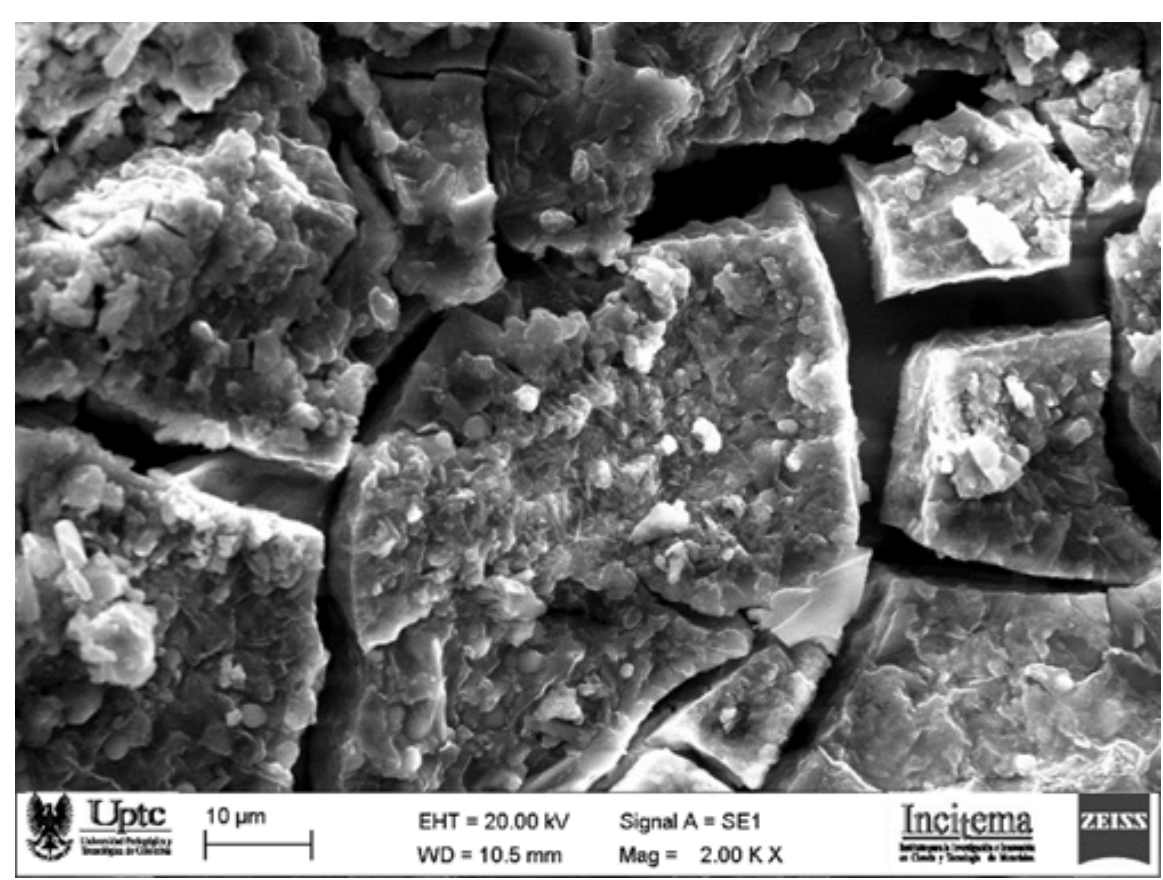

Fig. 10. Micrografía MEB mortero de escoria activado con $\mathrm{NA}_{2} \mathrm{SiO}_{3} / \mathrm{NaOH}$.

Fuente: Autores.

En la Fig. 11 se puede observar la microestructura de un mortero de escoria activado alcalinamente, con un zoom de 90um, en la cual se hizo el análisis del espectro para corroborar los elementos presentes en el material.

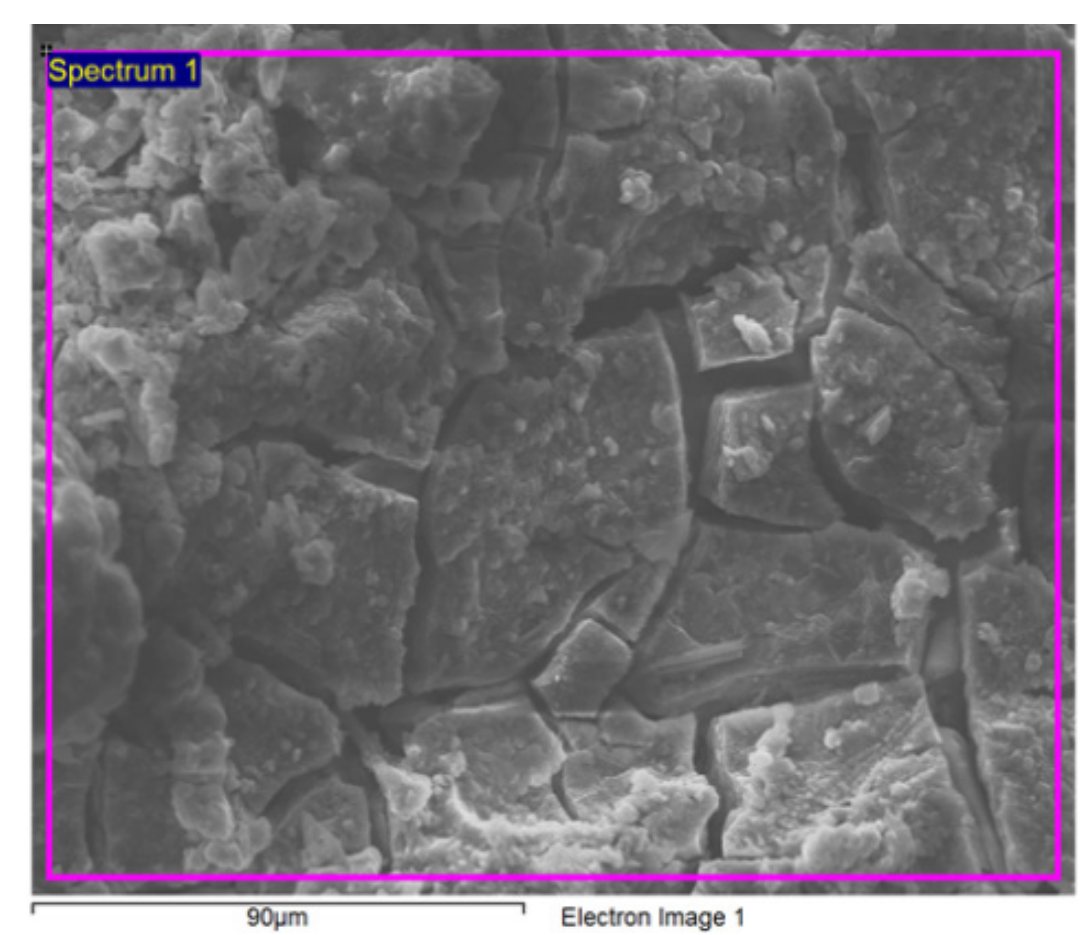

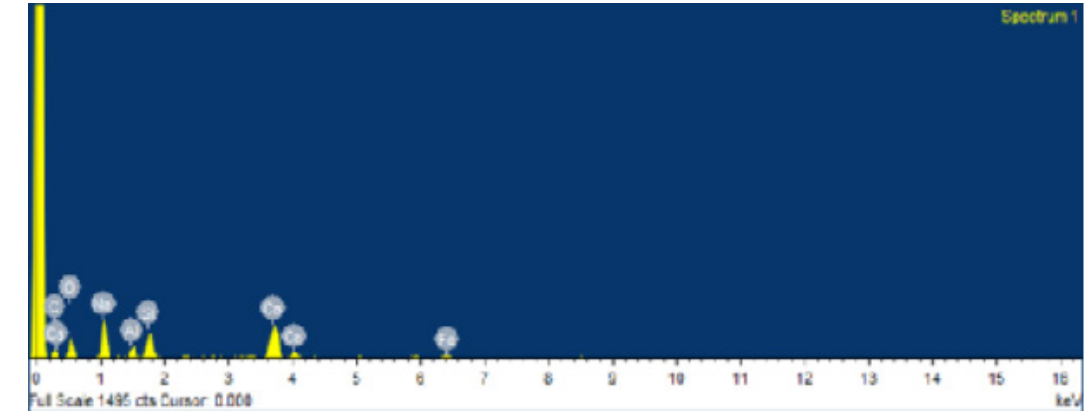

Fig. 11. Micrografías MEB y espectro EDX.

Fuente: Autores.

De las diferentes muestras observadas (Fig. 10 y Fig. 11) se deduce que en estos puntos no hay variación de la composición, tan solo presenta diferencia en el pico de $\mathrm{Na}=12.82 \%$, en peso, como consecuencia de la interacción entre la escoria y la disolución alcalina.

\section{Conclusiones}

La escoria con la cual se trabajaba estaba compuesta principalmente de $\mathrm{CaO}$ y $\mathrm{SiO}_{2}$. La escoria presentaba una estructura amorfa casi en su totalidad, la fase cristalina presente en la escoria sin activar es Gelenite y en la escoria activada con $\mathrm{Na}_{2} \mathrm{SiO}_{3} / \mathrm{NaOH}$ fue de andradita, el cual es un mineral del grupo de silicatos esto gracias a la reacción de activación.

Los porcentajes de activadores que brindaron mejor resultado a temperatura ambiente fueron 40\%, 50\% y $60 \%$, para los activadores Na2SiO3, Na2SiO3/ Na2SiO3 y Na2CO3/ Na2SiO3 respectivamente. Y los porcentajes de activadores con mejor resultado a temperatura de $60^{\circ} \mathrm{C}$ fueron también $40 \%, 50 \%$ y $60 \%$, para los activadores $\mathrm{Na}_{2} \mathrm{SiO}_{3}, \mathrm{Na}_{2} \mathrm{SiO}_{3} / \mathrm{NaOH}$ y $\mathrm{Na}_{2} \mathrm{SiO}_{3} /$ $\mathrm{Na}_{2} \mathrm{SiO}_{3}$ respectivamente.

$\mathrm{El} \mathrm{Na} \mathrm{CO}_{3} / \mathrm{Na}_{2} \mathrm{SiO}_{3}$ presenta el mejor comportamiento a la compresión en los sistemas preparados. Las pastas de $\mathrm{Na}_{2} \mathrm{SiO}_{3} / \mathrm{NaOH}$ y $\mathrm{Na}_{2} \mathrm{SiO}_{3} / \mathrm{Na}_{2} \mathrm{CO}_{3}$ sometidas a curado de $60^{\circ} \mathrm{C}$ presentaron resistencias inferiores con relación a las pastas curadas a temperatura ambiente.

El sistema que con las menores resistencias fue el $\mathrm{Na}_{2} \mathrm{SiO}_{3}$. Los morteros activados con $\mathrm{Na}_{2} \mathrm{SiO}_{3} / \mathrm{NaOH}$, presentaron el mejor comportamiento a compresión. Y los morteros activados con $\mathrm{Na}_{2} \mathrm{SiO}_{3}$, arrojaron los resultados más bajos de resistencia a la compresión. La inclusión de caliza en los morteros da como resultado la disminución en la resistencia de compresión.

De acuerdo a los resultados de la Tabla XI los morteros que presentaron mejor comportamiento fueron aquellos sometidos a temperaturas de $60^{\circ} \mathrm{C}$ y a mayor tiempo de curado aumentando sus propiedades de resistencia. Los morteros activados con $\mathrm{Na}_{2} \mathrm{SiO}_{3} / \mathrm{NaOH}$ sin incorporación de caliza alcanzaron el 93\% de su resistencia máxima a los 28 días a la edad 3 días.

Los morteros activados con $\mathrm{Na}_{2} \mathrm{SiO}_{3} / \mathrm{NaOH}$ con incorporación de caliza alcanzaron el $89 \%$ de su resistencia máxima a los 28 días a la edad de 3 días. 


\section{Financiamiento}

Artículo de investigación científica derivado del proyecto de investigación "Efecto del tipo de activador en un mortero de escoria mezclado con caliza activado alcalinamente", financiado por la "Universidad Pedagógica y tecnológica de Colombia en conjunto con el grupo de Investigación de ingeniería civil y ambiental (GICA). Año de inicio: 2016, año de finalización: 2017.

\section{REFERENCIAS}

[1] J. Martirena, P. Seijo, S. Kennas, I. Machado \& R. González, "Waste to energy technologies targeting the poor. The Cuba case study", in World Renewable Energy Congress 2003, Germany, Koeln. Jun. 29- Jul. 5, 2003.

[2] F. Puertas, M. Palacios \& R. de Gutiérrez, "Morteros de escoria activada alcalinamente. Propiedades y durabilidad". in 2 Congresso Nacional de Argamassas de Construcao, Lisboa, Portugal, Nov. 22-23, 2007.

[3] DANE. (2017, julio). Estadísticas de Cemento Gris - ECG [Online]. Available from https://www.dane.gov.co/index. php/estadisticas-por-tema/construccion/estadisticas-de-cemento-gris

[4] S. Ramos, S. Oreggioni, O. Baglivo, M. Domínguez \& D. Costoya, "Transformando subprodutos en nuevas materias primas-avances en argentina respecto de la reutilización de subproducos siderúrgicos", in Reducao Directa Minério de Ferro y Tecnologia Mineral, Vila Velha, Brasil, p. 741750, 2011.

[5] A. Purdon, "The action of alkalis on Black-fumance", $J$. Soc. Chem, vol. 59, no. 9, pp. 191-202, 1949.

[6] V. Glukhovsky, G. Rostovskaja \& G. Rumyna, "High straigth slag-alkaline cements", in 7Th Intern. Congr. Chem. Cem. Paris, Francia, 1980.

[7] V. Glukhovskij, Y. Zaitsev \& V. Pakhomov, "Slag-alkaline cements and concretes-structure, properties, technological and economical aspects of the use". Silicates industriels, vol. 48 , no. 10 , pp. 197-200, 1983.

[8] O. Gjorv, "Alkali Activation of a Norwegian Granulated Blast-Furnace Slag", in Third International Conference on the use of Fly Ash, Silica Fume, Slag \& Natural Pozzolans in Concrete, Trondheim, Norway, vol 2, SP-114, pp. 15011518. Jan, 1989.

[9] T. Kutti, "Hydration products of alkali activated slag", in 9 th International congress on chemistry of cements, New Delhi, India, pp. 468-474, 1992.

[10] J. Metso, "The alkali reaction of alkali-activated Finnish blast furnace slag", Silic. Ind, vol. 47, no. 4-5, pp. 123-127. 1982.

[11] P. Parameswaran \& A. Chatterjee, "Alkali activation of Indian blast furnace slag", in 8th International congress of chemical cement, Rio de Janeiro, Brazil, pp. 86-91, 1986.

[12] K. Isozaki, S. Iwamoto \& K. Nakagawa, "Some properties of alkali-activated slag cements", CAJ Rev, vol. 506, pp. 120-123, Jan. 1986.

[13] C. Shi, X. Wu \& M. Tang, "Research on alkali-activated cementitious systems in China: a review", Advances in Cement Research, vol. 5, no. 17, pp. 1-7, jan. 1993. https://doi. org/10.1680/adcr.1993.5.17.1

[14] S.-d. Wang, "Review of recent research on alkali-activated concrete in China", Magazine of Concrete Research, vol. 43, no. 154, pp. 29-35. mar. 1991. https://doi.org/10.1680/ macr.1991.43.154.29

[15] W.J. Clarke \& M. Helal, "Alkali activated slag and Portland/slag ultrafine cement", MRS Proceeding, vol. 179, pp. 219-232, 1989. https://doi.org/10.1557/PROC-179-219
[16] C. Shi, D. Roy \& P. Krivenko, Alkali-activated cements and concretes, London: Imprint CRC Press, 2003. https://doi. org/10.4324/9780203390672

[17] A. Fernández-Jiménez \& F. Puertas, "Influencia de la Concentración del Activador Sobre la Cinética del Proceso de Activación Alcalina de una Escoria de Alto Homo", Materiales de Construcción, vol. 47, no. 246, pp. 31-42. 1997. https://doi.org/10.3989/mc.1997.v47.i246.505

[18] E. Rodríguez, "Eficiencia de activadores alcalinos basados en diferentes fuentes de sílice para la producción de sistemas geopoliméricos de ceniza volante", Ingeniería de la Construcción y Proyectos de Ingeniería Civil, Universidad Politécnica de Valencia, España, Trabajo de investigación CST/MIH-05, Nov, 2009.

[19] L. Espinosa \& J. Escalante, "Morteros a base de vidrio de desecho/escoria de alto horno; activación mecanoquímica del vidrio en soluciones alcalinas", Nexo, vol. 24, no. 2, pp. 92-103. Mar. 2012. https://doi.org/10.5377/nexo.v24i2.657

[20] G. Kovalchuk, A. Fernández-Jiménez \& A. Palomo, "Alkali-activated fly ash: effect of thermal curing conditions on mechanical and microstructural development-Part II". Fuel, vol. 86, no. 3, pp. 315-322. Feb. 2007. https://doi. org/10.1016/j.fuel.2006.07.010

[21] Determinación de la resistencia de morteros de cemento hidráulico usando cubos de 50mm o 50,8mm de lado, Icontec NTC 220, 1998.

[22] P. Krivenko, "Alkaline cements: terminology, classification, aspects of durability", in Congress on the chemistry of cements, Kiev, Ukraine, Jun. 25-27, 1997.

Nelson Virgilio Torres Rubio es Ingeniero Metalúrgico de la Universidad Pedagógica y Tecnológica (Colombia). Actualmente investigador en el grupo de investigación de ingeniería civil y ambiental (GICA) y del semillero multidisciplinar de la facultad de ingeniería (SOFIA) de la Universidad Pedagógica y Tecnológica (Colombia). https://orcid.org/0000-0002-9117-9434

Jorge Andrés Sarmiento-Rojas es Ingeniero Civil con Máster en Administración de Empresas (MBA) con énfasis en Finanzas y Máster en Gestión Integral de Proyectos, docente universitario a nivel de pregrado, con experiencia en investigación, procesos de identificación de mercado, diseño e implementación de estrategias comerciales, captación de nuevos clientes, gestión de proyectos, y en la construcción de obras civiles participa en los procesos de planificación, desarrollo, monitoreo y control. https://orcid.org/00000002-4230-3304

Oscar Javier Gutiérrez-Junco es Ingeniero Civil y especialista en Estructuras de la Universidad Nacional (Colombia) con Magister en Ingeniería Civil de la Universidad de los Andes (Colombia). Doctorado en Ingeniería y Ciencia de los Materiales de la Universidad Pedagógica y Tecnológica (Colombia). Actualmente es docente asociado de la UPTC, donde además ejerce los cargos de Coordinador Académico de Posgrados de Ingeniería Civil, Director del Centro de Gestión de Investigación y Extensión - CEDEC, y Director del Grupo de Investigación en Ingeniería Civil y Ambiental - GICA. http://orcid.org/0000-0002-5748-5032 